\title{
Agreement with employer influenza vaccination requirements among US healthcare personnel during the 2016-2017 season
}

\author{
Marie A. de Perio $\mathrm{MD}^{1}$, Xin Yue MPS, $\mathrm{MS}^{2}$, A. Scott Laney MPH, $\mathrm{PhD}^{3}$, Stacie M. Greby DVM, $\mathrm{MPH}^{4}$ and \\ Carla L. Black PhD ${ }^{4}$ \\ ${ }^{1}$ Division of Surveillance, Hazard Evaluations, and Field Studies, National Institute for Occupational Safety and Health, Centers for Disease Control and \\ Prevention, Cincinnati, Ohio, ${ }^{2}$ Leidos, Inc, Reston, Virginia, ${ }^{3}$ Respiratory Health Division, National Institute of Occupational Safety and Health, Centers for \\ Disease Control and Prevention, Morgantown, West Virginia and ${ }^{4}$ Immunization Services Division, National Center for Immunization and Respiratory Diseases, \\ Centers for Disease Control and Prevention, Atlanta, Georgia
}

To the Editor-Annual vaccination of healthcare personnel (HCP) is a high priority for reducing influenza-associated morbidity in healthcare settings. ${ }^{1}$ Although the percentage of HCP nationwide who reported receiving influenza vaccination was $78.6 \%$ in the $2016-$ 2017 season, coverage remains incomplete, placing HCP and patients at risk of influenza. ${ }^{1,2}$ Employer influenza vaccination requirements are associated with higher coverage rates, and though controversial, mandatory influenza vaccination is supported by multiple healthcare professional societies. ${ }^{3,4}$ We explored agreement with employer influenza vaccination requirements among HCP nationwide.

\section{Methods}

We used data from the opt-in Internet panel survey of HCP for the 2016-2017 influenza season conducted from March 28 to April 19, 2017. Similar surveys have been conducted using 2 national opt-in Internet sources since the 2010-2011 influenza season to provide estimates of influenza vaccination coverage among HCP. ${ }^{2}$

In addition to questions about influenza vaccination coverage, occupation type, and healthcare setting, the survey included the statement, "Healthcare workers should be required to be vaccinated for flu." Respondents were asked to rate their agreement with the statement using a 4-point scale. Responses of strongly agree and agree were combined and categorized as expressed agreement. Responses of strongly disagree and disagree were combined and categorized as expressed disagreement.

We weighted responses to the US HCP population by age, sex, race/ethnicity, work setting, and census region based on Bureau of Labor Statistics and US Census Bureau data. We calculated the number and weighted percentage of HCP who expressed agreement or disagreement with the vaccination requirement statement by occupation type, healthcare setting, vaccination status, and employer promotion efforts. Additional statistical analyses were not performed because this survey included an opt-in sample and a nonrandom sample.

\section{Results}

Overall, $72.9 \%$ of 2,438 respondents expressed agreement with the vaccination requirement statement. In total, $78.6 \%$ of respondents

Author for correspondence: Marie A. de Perio, MD, National Institute for Occupational Safety and Health, Centers for Disease Control and Prevention, 1090 Tusculum Ave, R-9, Cincinnati, OH 45226. E-mail: mdeperio@cdc.gov

Cite this article: de Perio MA, et al. (2018). Agreement With Employer Influenza Vaccination Requirements Among US Healthcare Personnel During the 2016-2017 Season. Infection Control \& Hospital Epidemiology 2018, 39, 1019-1020. doi: 10.1017/ ice.2018.111

(c) 2018 by The Society for Healthcare Epidemiology of America. All rights reserved. reported receiving an influenza vaccination during the 2016-2017 season, and $42.3 \%$ of respondents reported working in a setting with employer influenza vaccination requirements. ${ }^{2}$ Table 1 shows the weighted percentage of respondents who expressed agreement with the statement by occupation type, healthcare setting, vaccination status, and employer promotion efforts. By healthcare setting, the weighted percentage of respondents who expressed agreement ranged from 70.9\% (ambulatory care settings) to $77.2 \%$ (hospitals). By occupation, the weighted percentage of respondents who expressed agreement ranged from $67.4 \%$ (other clinical HCP) to $85.7 \%$ (physicians).

By influenza vaccination status, the weighted percentage of respondents who expressed agreement with the vaccination requirement statement was $83.5 \%$ for those who received the vaccine and $33.9 \%$ for those who did not. Of those who agreed with the vaccination requirement statement yet did not receive it, the most common reasons given for not getting it were "I haven't gotten around to it (18.0\%)," "I just don't want the vaccine (15.7\%)," and "My employer didn't require me to have a vaccination (14.1\%)." By workplace vaccination promotion, the weighted percentage of respondents who expressed agreement ranged from $56.4 \%$ for those with no employer requirement, onsite vaccination, or promotion to $81.6 \%$ for those with an employer vaccination requirement.

\section{Discussion}

Mandatory influenza vaccination is increasingly common in healthcare settings, and multiple states have established influenza vaccination requirements for hospital HCP. ${ }^{5}$ However, concerns have been raised related to the variable effectiveness of the vaccine and the ethical and legal impact of these policies. ${ }^{5,6}$ The duty of a HCP to protect the health of individual patients and the public competes with their right to personal autonomy. Mandates also invoke legal issues, including the applicability of state and federal constitutional laws and statutes.

Nevertheless, in our study, most HCP (72.9\%) from all occupational groups and healthcare settings agreed with employer vaccination requirements. These findings suggest an increase when compared with a 2010 online research panel of HCP nationwide, showing that $57.4 \%$ of HCP agreed with influenza vaccination requirements. Implementation of employer vaccination requirements is becoming more common ( $11.1 \%$ of respondents in 2010 compared with $42.3 \%$ in our study). ${ }^{4}$ This finding is similar to those of other nationally representative samples among hospitals $(43 \%-44 \%)$. ${ }^{8,9}$

In our study, employees in hospitals, ambulatory care, and long-term care settings had similar agreement with employer vaccination requirements $(70.9 \%-77.2 \%)$. However, physicians, 
Table 1. Percentage of Healthcare Personnel (HCP) Who Expressed Agreement With the Statement, "Healthcare Workers Should Be Required To Be Vaccinated For Flu" in an Internet Panel Survey Conducted in the United States During the 2016-2017 Influenza Season

\begin{tabular}{|c|c|}
\hline Variable & $\begin{array}{l}\text { No. Who Expressed } \\
\text { Agreement (Weighted } \%^{a} \text { ) }\end{array}$ \\
\hline Overall & $1,810(72.9)$ \\
\hline \multicolumn{2}{|l|}{ Healthcare setting ${ }^{\mathrm{b}}$} \\
\hline Hospital & $925(77.2)$ \\
\hline Ambulatory care & $718(70.9)$ \\
\hline Long-term care & $549(71.0)$ \\
\hline Other setting ${ }^{c}$ & $604(73.4)$ \\
\hline \multicolumn{2}{|l|}{ Occupation } \\
\hline Physician & $251(85.7)$ \\
\hline Nurse practitioner/Physician assistant & $154(81.0)$ \\
\hline Nurse & $167(73.7)$ \\
\hline Pharmacist & $307(80.6)$ \\
\hline Assistant/aide & $641(69.9)$ \\
\hline Other clinical HCP ${ }^{d}$ & $572(67.4)$ \\
\hline Nonclinical $\mathrm{HCP}^{\mathrm{e}}$ & $315(75.7)$ \\
\hline \multicolumn{2}{|l|}{ Influenza vaccination receipt } \\
\hline Received influenza vaccination & $1,989(83.5)$ \\
\hline Did not receive influenza vaccination & $449(33.9)$ \\
\hline \multicolumn{2}{|l|}{ Workplace vaccination promotion } \\
\hline Employer vaccination requirement ${ }^{f}$ & $983(81.6)$ \\
\hline Onsite vaccination ${ }^{\mathrm{g}}$ & $795(70.1)$ \\
\hline Other vaccination promotion ${ }^{\mathrm{h}}$ & $206(78.6)$ \\
\hline $\begin{array}{l}\text { No requirement, onsite vaccination, or } \\
\text { promotion }\end{array}$ & $454(56.4)$ \\
\hline
\end{tabular}

${ }^{a}$ Weights were calculated based on each occupation type, by age, sex, race/ethnicity, healthcare setting, and US census region to represent the US HCP population.

${ }^{\mathrm{b}}$ Respondents could choose $>1$ setting.

${ }^{c}$ Dentist office or dental clinic, pharmacy, laboratory, public health setting, emergency medical services setting, or other setting where clinical care or related services was provided to patients.

${ }^{\mathrm{d}}$ Allied health professional, technician, or technologist.

${ }^{\mathrm{e}}$ Administrative support staff members or manager and nonclinical support staff members including food service workers, laundry workers, janitors, and members of the housekeeping and maintenance staff.

fIncludes all respondents who indicated that their employer required them to be vaccinated for influenza.

${ }^{g}$ Employer made influenza vaccination available on-site for at least 1 day during the influenza season at no cost to employees. Restricted to respondents without an employer requirement for vaccination.

hInfluenza vaccination was promoted among employees through public identification of vaccinated persons, financial incentives or rewards to individuals or groups of employees, competition between units or care areas, free or subsidized cost of vaccination, personal reminders to be vaccinated, publicizing the number or percentage of employees receiving vaccination, or making vaccination available at special events organized on site. Restricted to respondents without an employer requirement for vaccination or on-site vaccination.

pharmacists, and nurse practitioners/physician assistants had the highest agreement with employer vaccination requirements (all $>80 \%$ ), and other clinical HCP (67.4\%) and assistants/aides (69.9\%) had the lowest agreement.
Nearly one-third of HCP not vaccinated agreed with the employer influenza vaccination requirement statement. Also, $56.4 \%$ of HCP work in settings without vaccination requirement, onsite vaccination, or promotion efforts in combination with vaccination requirements. These findings suggest that employer vaccination requirements, particularly in long-term care settings, could increase influenza vaccination coverage.

Notably, the survey question lacked details on what "required" vaccination entails. However, vaccine requirement is generally defined as any institutional policy that requires HCP to receive influenza vaccine or decline to receive one, with or without consequences for vaccine refusal. ${ }^{10}$ Examples of consequences include termination, mask wearing, and restriction from patient care duties. Exploring the consequences was outside the scope of this study.

Most HCP from all occupational groups and healthcare settings agreed with vaccination requirements, including $33.9 \%$ of those not vaccinated. Wider adoption of employer vaccination requirements in all healthcare settings has support, can increase HCP influenza vaccination, and may reduce influenza transmission in healthcare settings. Interventions known to improve coverage, such as educating workers and offering vaccine on site, at no cost, and during work hours should be implemented.

Acknowledgments. We acknowledge the staff of Abt Associates for their contributions to data collection. The findings and conclusions in this manuscript are those of the authors and do not necessarily represent the official position of the US Centers for Disease Control and Prevention.

Financial support. No financial support was provided relevant to this article.

Potential conflicts of interest. All authors report no conflicts of interest relevant to this article.

\section{References}

1. CDC. Immunization of health-care personnel: recommendations of the Advisory Committee on Immunization Practices (ACIP). MMWR Recom Rep 2011;60(No. RR-07.

2. Black CL, Yue C, Ball SW, et al. Influenza vaccination coverage among health care personnel-United States, 2016-17 influenza season. MMWR Morb Mortal Wkly Rep 2017;66:1009-1015.

3. Pitts SI, Maruthur NM, Millar KR, Perl TM, Segal J. A systematic review of mandatory influenza vaccination in healthcare personnel. Am J Prev Med 2014;47:330-340.

4. Maurer J, Harris KM, Black CL, Euler GL. Support for seasonal influenza vaccination requirements among US healthcare personnel. Infect Control Hosp Epidemiol 2012;33:213-221.

5. Stewart AM, Caplan A, Cox MA, Chang KHM, Miller JE. Mandatory vaccination of health-care personnel: good policy, law, and outcomes. Jurimetrics 2013;53:341-359.

6. Quan K, Tehrani DM, Dickey L, et al. Voluntary to mandatory: evolution of strategies and attitudes toward influenza vaccination of healthcare personnel. Infect Control Hosp Epidemiol 2012;33:63-70.

7. Randall LH, Curran EA, Omer SB. Legal considerations surrounding mandatory influenza vaccination for healthcare workers in the United States. Vaccine 2013;31:1771-1776.

8. Miller BL, Ahmed F, Lindley MC, Wortley PM. Increases in vaccination coverage of healthcare personnel following institutional requirements for influenza vaccination: a national survey of US hospitals. Vaccine 2011;29:9398-9403.

9. Greene MT, Fowler K, Krein SL, et al. Influenza vaccination requirements for healthcare personnel in US hospitals: results of a national survey. Infect Control Hosp Epidemiol 2016;37:485-487. 